\title{
Correction: PPA1 promotes NSCLC progression via a JNK- and TP53-dependent manner
}

Dehong Luo, Daishun Liu, Wen Shi, Huimin Jiang, Wei Liu, Xiaoyuan Zhang, Yonghua Bao, Wancai Yang, Xiaojun Wang, Chaoyang Zhang, Hui Wang, Liying Yuan, Yanpei Chen, Tianyin Qu, Dong Ou, Wenzhi Shen and Shuang Yang

\section{Correction to: Oncogenesis}

https://doi.org/10.1038/s41389-019-0162-y

published online 24 September 2019
The wrong western blot data was used in Fig. $2 \mathrm{~d}$ and Fig. $3 \mathrm{~d}$ in this Article. The correct versions of the figures are provided below. The scientific conclusions of this paper were not affected.

Published online: 13 December 2019 


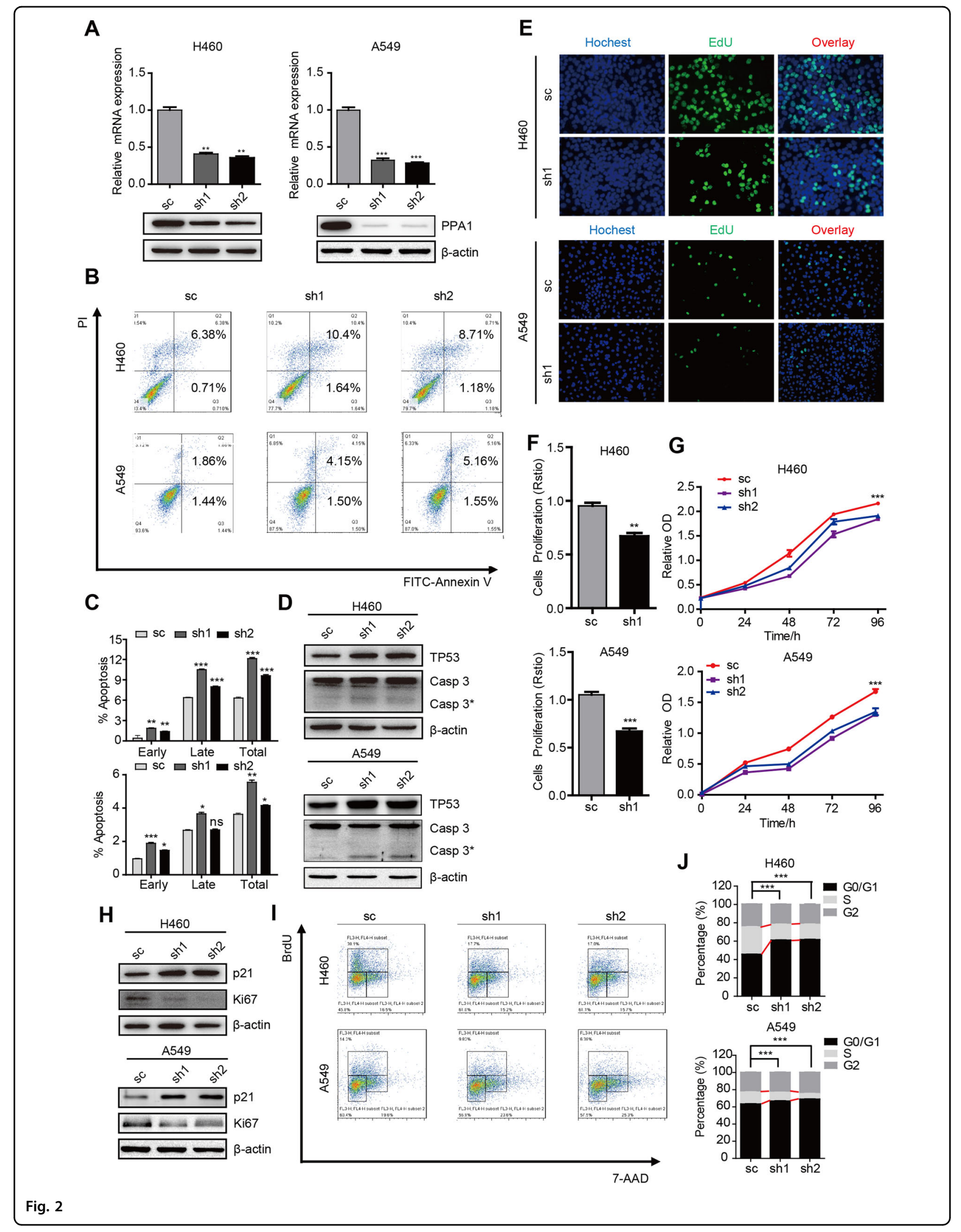




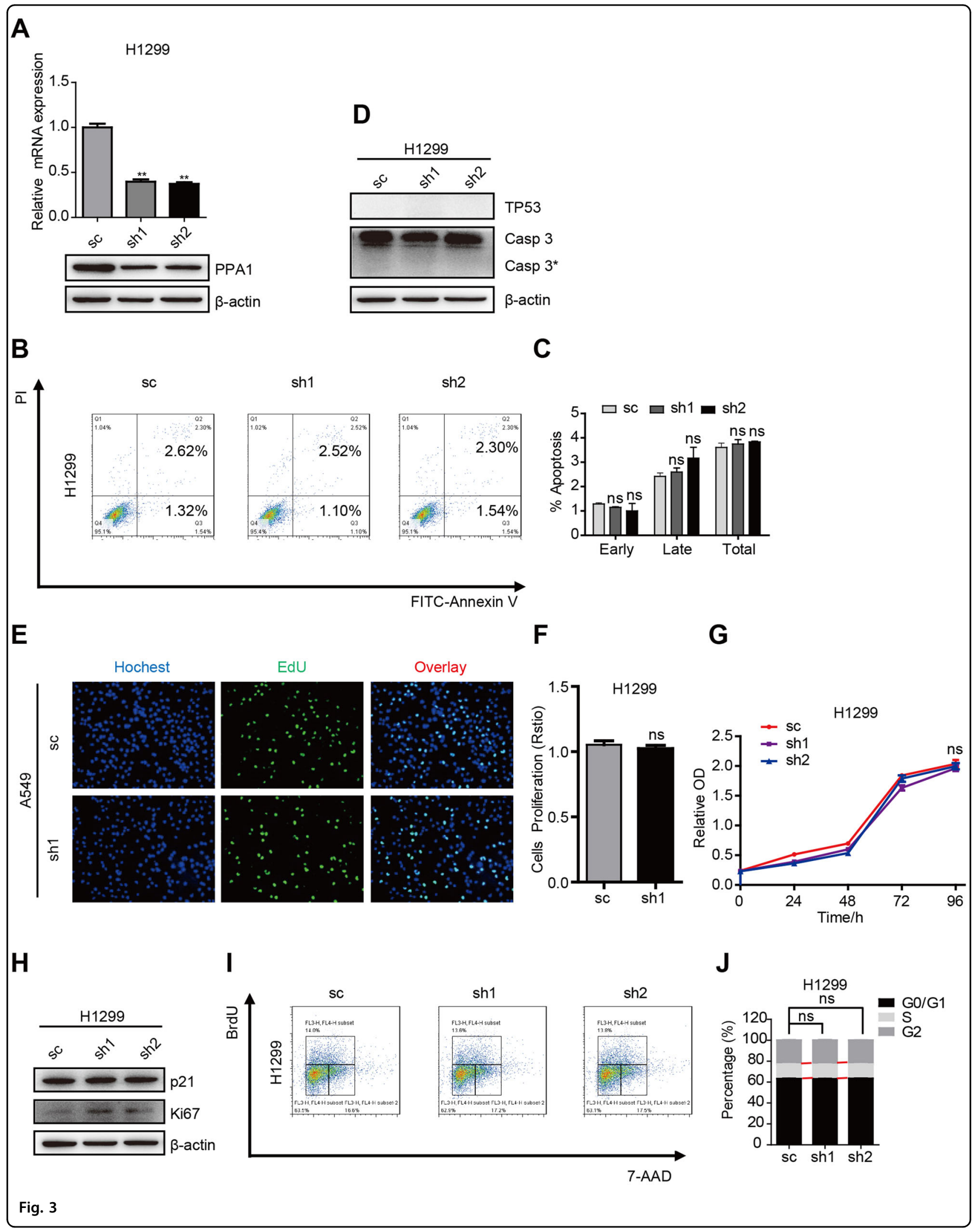

